Electronic Supplementary Information

\title{
Graphitic Carbon Coated Magnetite Nanoparticles for Dual Mode Imaging and Hyperthermia
}

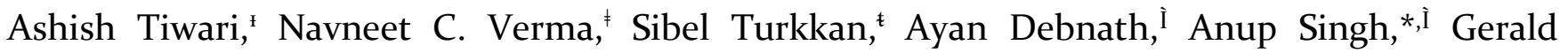
Draeger $^{*, \pm}$, Chayan K. Nandi ${ }^{*, \neq}$ and Jaspreet K. Randhawa*,

'School of Engineering, Indian Institute of Technology Mandi, Mandi, Himachal Pradesh-175005, India

${ }^{\ddagger}$ School of Basic Sciences, Indian Institute of Technology Mandi, Mandi, Himachal Pradesh-175005, India

${ }^{t}$ Leibniz University of Hannover, Institut für Organische Chemie Schneiderberg, Schneiderberg $1 \mathrm{~b}$, 30167-Hannover, Germany

İCenter for BioMedical Engineering, Indian Institute of Technology Delhi, Hauz Khas, New

Delhi-110016, India

*Corresponding author postal address

Dr. Jaspreet Kaur Randhawa

School of Engineering,

Indian Institute of Technology Mandi, Mandi-175005, India

Telephone: +91-1905-267056

Email: jaspreet@iitmandi.ac.in 


\section{Table of content}

\section{Figures, Tables and Videos}

Photo S1. Experimental set up for the magnetic hyperthermia measurements comprised of three main components: the power supply, an external capacitive network and an induction coil.

Table T1. Synthesis parameters for the preparation of SPIONs.

Table T2: The size of primary magnetite nanoparticles in all the three SPIONs measured using HRTEM and the mean crystalline size of magnetite nanoparticles calculated from XRD results using Scherrer equation.

Figure S1. TEM images of SPIONs showing the particle size and morphology with the clear core-shell structures.

Figure S2. EDAX spectra images of (a) $40 \mathrm{~nm}$ carbon coated SPIONs (b) $10 \mathrm{~nm}$ carbon coated SPIONs and (c) carbon composite SPIONs respectively.

Figure S3. XRD spectra (a-c), full survey XPS spectra (d-f), FTIR spectra (g-i) and Raman spectra (j-l) of $40 \mathrm{~nm}$ carbon coated SPIONs, $10 \mathrm{~nm}$ carbon coated SPIONs and carbon composite SPIONs respectively.

Figure S4. High-resolution deconvoluted Fe2p XPS spectra of SPIONs displayed two distinct peaks correspond to $\mathrm{Fe} 2 \mathrm{p}_{3 / 2}$ and $\mathrm{Fe} 2 \mathrm{p}_{1 / 2}$ which were deconvoluted into four peaks along with one shakeup satellite peak. (a) $40 \mathrm{~nm}$ carbon coated SPIONs (b) $10 \mathrm{~nm}$ carbon coated SPIONs and (c) carbon composite SPIONs respectively.

Figure S5. High-resolution deconvoluted C1s XPS spectra of SPIONs showed three peaks for C=C, C$\mathrm{OH}$ and $\mathrm{C}=\mathrm{O}$ respectively. (a) $40 \mathrm{~nm}$ carbon coated SPIONs (b) $10 \mathrm{~nm}$ carbon coated SPIONs and (c) carbon composite SPIONs respectively.

Figure S6. High-resolution deconvoluted O1s spectra of SPIONs exhibited three peaks correspond to lattice oxygen of $\mathrm{Fe}_{3} \mathrm{O}_{4}$, carbonyl oxygen in $\mathrm{O}-\mathrm{C}=\mathrm{O}$ and $\mathrm{C}-\mathrm{O}$ groups respectively. (a) $40 \mathrm{~nm}$ carbon coated SPIONs (b) $10 \mathrm{~nm}$ carbon coated SPIONs and (c) carbon composite SPIONs respectively.

Figure S7. Emission spectra of SPIONs in bulk solution excited at $370 \mathrm{~nm}$ (a) $40 \mathrm{~nm}$ carbon coated SPIONs (b) $10 \mathrm{~nm}$ carbon coated SPIONs and (c) carbon composite SPIONs respectively. 
Figure S8. Photoluminescence Raman spectra of SPIONs before and after annealing the sample upto 300 ${ }^{\circ} \mathrm{C}$ in air which display the disappearance of luminescence peaks in annealed samples. (a) $40 \mathrm{~nm}$ carbon coated SPIONs (b) $10 \mathrm{~nm}$ carbon coated SPIONs and (c) carbon composite SPIONs respectively.

Figure S9. Plot of R2 or 1/T2 vs. Fe concentration of SPIONs. (a) $40 \mathrm{~nm}$ carbon coated SPIONs (b) 10 $\mathrm{nm}$ carbon coated SPIONs and (c) carbon composite SPIONs respectively. The red line in the plots represents a linear fit.

Figure S10. The heating profile curves of samples (a) $40 \mathrm{~nm}$ carbon coated SPIONs, (b) $10 \mathrm{~nm}$ carbon coated SPIONs and (c) carbon composite SPIONs $(2 \mathrm{mg} / \mathrm{ml})$ in different magnetic field amplitudes (10, 15,20 and $30 \mathrm{kA} / \mathrm{m}$ ) at the frequency of $150 \mathrm{kHz}$.

Table T3. X-ray spectroscopy analysis of the SPIONs.

Table T4. Functional group analysis of the SPIONs.

Table T5. Magnetic hyperthermia results with increase in temperature and SLP values.

Video SV1. Real time single particle fluorescence microscopy video of all the three SPIONs. 


\section{Materials}

All the chemicals, ferrocene, hydrogen peroxide and phosphate buffer solution (PBS) were purchased from Sigma Aldrich. Solvents used in synthesis were of analytical grade. All chemicals were used without further purification.

\section{Synthesis of SPIONs nanostructures}

In a typical synthesis, ferrocene $(0.20 \mathrm{~g})$ was dissolved in acetone solution $(60 \mathrm{~mL})$. After intense sonication for 1 hour, $5 \mathrm{~mL}$ of hydrogen peroxide solution was slowly added to the ferrocene solution, which was then vigorously stirred for another 30 minutes under magnetic stirring. The solution was then transferred to a $100 \mathrm{~mL}$ Teflon-lined stainless steel autoclave. After sealing, the autoclave was heated at $220^{\circ} \mathrm{C}$ for 48 hours. The autoclave was then cooled naturally at room temperature. The supernatant was carefully discarded using a magnet. Further, the precipitate was then washed with acetone three times. Finally, the black precipitate was dried at $30^{\circ} \mathrm{C}$ in a vacuum oven and used for further characterization.

\section{Characterization}

Powder X-ray diffraction (PXRD) pattern was recorded on a Rigaku Smart Lab diffractometer, using $\mathrm{CuK} \alpha$ radiation from 5 to 80 degree $2 \theta$ with scanning rate of 2 degree $/ \mathrm{min}$. The particle morphology and elemental mapping of SPIONs samples were obtained by transmission electron microscopy (TEM) using FEI Tecnai TEM equipped with a LaB6 source operating at $200 \mathrm{kV}$. Scanning transmission electron microscopy-high angle annular dark field (STEM-HAADF) imaging was also performed on the TEM. The morphology and energy dispersive $\mathrm{x}$-ray analysis (EDS) of SPIONSs were observed using a field emission scanning electron microscopy FESEM (NOVA NanoSEM 450) at an accelerating voltage of $10 \mathrm{kV}$. Fluorescence spectra were recorded on Horiba spectrophotometer from wavelength range of 200 to $800 \mathrm{~nm}$. The average lifetime of SPIONs was calculated from fluorescence lifetime decay measurements using excitation wavelength of $380 \mathrm{~nm}$ and emission wavelength of $430 \mathrm{~nm}$. Fourier transformed infrared (FTIR) 
spectra were recorded with Agilent Technologies Cary 6000 series FTIR spectrometer at wavenumber from 400 to $4000 \mathrm{~cm}^{-1}$. Magnetic properties were analysed through vibrating sample magnetometer-superconducting quantum interference device (VSM-SQUID) under the vibrating magnetic field of $4 \mathrm{~T}$ at $300 \mathrm{~K}$. FC and ZFC analysis was similarly performed on the SQUIDVSM. X-ray photoelectron spectra (XPS) was performed on a VG ESCALAB250 electron spectrometer with a monochromatic $\mathrm{Al} \mathrm{K} \alpha(1486.6 \mathrm{eV})$ at $15 \mathrm{kV}$ and $10 \mathrm{~mA}$, and all binding energies were referenced to the $\mathrm{C} 1 \mathrm{~s}$ peak $(284.0 \mathrm{eV})$ prior to the data analysis. Raman experiments were performed on a Horiba LABRAM high-resolution Raman spectrophotometer using $534 \mathrm{~nm}$ He-Ne laser with exposure time of 30s. Photoluminescence Raman spectra were also recorded on the same instruments by heating the sample upto $300{ }^{\circ} \mathrm{C}$ from the wavelength range of 550 to 750 nm.

\section{Phantom Preparation for Magnetic resonance imaging (MRI) scanning}

For MRI scanning, base solution with $1.5 \%$ agarose was prepared. This base solution was put into a large (2L) cylindrical beaker as well as in nine $15 \mathrm{~mm}$ small test tubes. In small test tubes different iron concentration were added to get concentrations of $0.005 \mathrm{mM}, 0.01 \mathrm{mM}, 0.015 \mathrm{mM}, 0.02 \mathrm{mM}, 0.03 \mathrm{mM}$, $0.04 \mathrm{mM}, 0.05 \mathrm{mM}, 0.1 \mathrm{mM}, 0.25 \mathrm{mM}, 0.5 \mathrm{mM}$ respectively. These small phantoms, 1 to 9 , were inserted vertically (circumscribed) inside large phantom.

\section{MR imaging and relaxivity studies of SPIONs}

MRI experiments were performed at 3T whole-body MRI system (Ingenia, Philips Healthcare, The Netherlands) using a 16 channel receive only coil. Data for T2 map was acquired using vendor supplied T2 mapping pulse sequence with option of multiple echoes. MRI protocol consisted of FOV=200*200, matrix size $=256^{*} 256$, slice thickness $=6 \mathrm{~mm}$, number of slices $=12, \mathrm{TR}=6000 \mathrm{~ms}$ and $\mathrm{TE}=30,60,90$, 120, 150, 180, $210 \mathrm{~ms}$. MRI data for T1 map was also acquired using inversion recovery based pulse sequence. For this sequence we used TI $=100,300,500,700,1000,1500,2000,2500,3000$ and 3500 ms. 


\section{Relaxivity mapping}

MRI images were processed using in house written programs in MATLAB. Voxelwise $\mathrm{T}_{2}$ map was generated by fitting signal intensity data corresponding to different TEs to following mono-exponentially decaying function:

$$
S(T E)=A \times e^{-\frac{T E}{T_{2}}}
$$

ROIs were drawn over small phantoms and average $\mathrm{T} 2$ values were computed for each ROI. R2 $=1 / \mathrm{T}_{2}$ values were computed. Transverse Relaxivity (r2) of contrast agent is estimated using following equation:

$$
R_{2}=R_{20}+r 2 \times C
$$

Where $R_{2}$ is concentration dependent relaxation time and $R_{20}$ is relaxation time of base solution or agarose without contrast agent.

\section{Single particle fluorescence imaging (time trace and photon counts) studies of SPIONs}

SPIONs were spin coated on a clean glass slide to analyse the single-particle time traces. The diffraction limited spots produced due to single-particle blinking were observed using $100 \times$ Nikon total internal reflection fluorescence (TIRF) objectives. For excitation, a $532 \mathrm{~nm}$ diode laser was used. An oil immersion Nikon TIRF objective (100× magnification and 1.49 NA) was mounted on a custom-built inverted optical microscope. To separate the excitation and emission light, a $532 \mathrm{~nm}$ high pass Dichroic (AHF Analysentechnik) was used for imaging. Further, to record the single particle photon counts, an Andor electron multiplication charge coupled device (EMCCD) iXon Ultra was used at a readout rate of $17 \mathrm{MHz}$ with exposure time $50 \mathrm{~ms}$. Time trajectories were analysed by Andor Solis Software. Typically, the incident photons were converted to electrons and subsequently counted by the EMCCD camera during imaging. In addition, the 'analyse region of interest (ROI)' tool of Andor solis was significantly used to extract the total counts along with the maximum, mean and standard deviation values using a group of pixels. The 
movies were recorded under the kinetic mode of the EMCCD. The time/frame trajectories of the counts/intensity at given pixel were obtained for the given exposure time to record the movies. These time trajectories were further used for analysis. The image area was taken as only $128 \times 128$ pixels from the total $512 \times 512$ pixels of the whole sensor of the EMCCD. Thus, we used a total image area of $64 \times 64$ pixels $(10.24 \mu \mathrm{m} \times 10.24 \mu \mathrm{m})$ with a pixel size of $160 \mathrm{~nm}$ using $100 \times$ magnification. The Andor iXon Ultra EMCCD camera has a pixel size of $16 \mu \mathrm{m} \times 16 \mu \mathrm{m}$, so on a system with a $100 \times$ objective lens with the pixel size of $160 \mathrm{~nm}(16 \mu \mathrm{m} / 100)$. During the measurement, the background was calculated from the image area where no particles were present. A background free signal was obtained after subtracting the mean value of the background from the original trajectory. The background signals (after complete photobleaching) were also set to zero level for further correction.

\section{Alternating magnetic field (AMF) equipment for hyperthermia studies}

The alternating magnetic field generator TruHeat HF series 500 AXIO 10/450T (Trumpf Hüttinger, Germany) is comprised of three main components: the power supply, an external capacitive network and an induction coil. The ensemble creates a resonant circuit. While in operation, the coil is cooled by closedloop circulating cooling water system. The five-turn copper pancake coil inductor (inner diameter: $52 \mathrm{~mm}$ ) is used to generate range of homogeneous magnetic field. The magnetic field amplitude at fixed frequency can easily be altered by modifying the power supply voltage. The frequency was adjusted by altering the capacitance of the external capacitive network, in regard the range of attainable frequency was $300-390 \mathrm{kHz}$. We performed heating experiments with intervals between 10 and $30 \mathrm{kA} / \mathrm{m}$ within $300-400 \mathrm{kHz}$. 

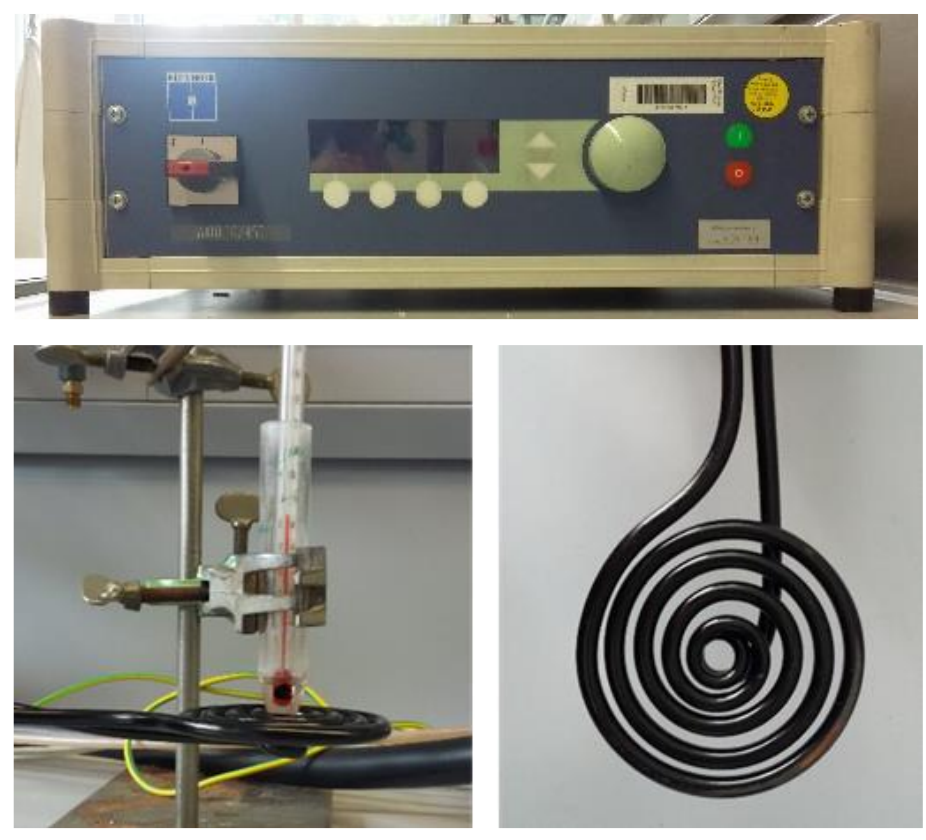

Photo S1. Experimental set up for the magnetic hyperthermia measurements comprised of three main components: the power supply, an external capacitive network and an induction coil.

\section{Specific loss power (SLP) measurements and calculations}

The specific loss power of nanoparticle sample is defined as the measured thermal loss power, normalized by mass of magnetic material with units (W/g magnetic material). 1-2 $\mathrm{mg} / \mathrm{ml}$ solution of magnetic nanoparticles was dispersed in a conical-bottom glass tube and placed in the center of the pancake coil. The temperature change was recorded by immersing a red spirit thermometer in aqueous magnetic nanoparticle suspension. The experimental setup is depicted in Fig.

$$
S L P(W / g)=\frac{m_{s} * c_{p}}{m_{n p}} * \frac{\Delta T}{\Delta \mathrm{t}}
$$

where $m_{s}$ is the mass of solution, $m_{n p}$ is the mass of superparamagnetic nanoparticles, $c_{p}$ is the heat capacity of the solution, and $(\Delta T / \Delta t)$ is the initial slope of temperature rise vs. time curve in heating profile. 


\section{Material synthesis}

Three different core-shell SPIONs have been prepared in a single step solvothermal method by varying the synthesis parameters as described in the supporting information (Table S1 SI). A magnetic core assembly of SPIONs nanocrystals coated with fluorescent carbon opted the spherical core-shell nanostructures. An attempt was made in situ to control the carbon coating over SPIONs which produced three different types of core-shell nanostructures. The synthesis was optimized by modulating the reaction parameters i.e. precursor concentration, amount of oxidizing agent and reaction temperature etc. A detail insight showed that modulation of synthesis parameters yield different core-shell nanostructures. Reaction temperature and amount of oxidizing agent made a strong impact on the morphology of core-shell nanostructures. In brief to understand the reaction mechanism of the synthesis of these three SPIONs, the following hypothesis is proposed. Initially, an eminent amount of ferrocene is dissolved in acetone followed by addition of $\mathrm{H}_{2} \mathrm{O}_{2}$ and subsequently heated in a sealed chamber resulting the decomposition of ferrocene into Fe cations and cyclopentadiene. Inside the sealed chamber at high temperature, cyclopentadiene starts decomposing which generates the carbon free radicals. Since, these newly form carbon radicals have high surface energies, they got agglomerated and subsequently form larger radicals by minimizing surface energies. Besides this process, $\mathrm{O}_{2}$ is generated by the decomposition of $\mathrm{H}_{2} \mathrm{O}_{2}$ which then oxidize Fe cations to form small iron oxide nanoclusters simultaneously. Meantime, the generated Fe cations then catalyze the $\mathrm{H}_{2} \mathrm{O}_{2}$ decomposition and generate new free carboxyl and hydroxyl radicals. These newly formed radicals accelerates the iron oxide formation and simultaneously form hydrophilic carboxyl and hydroxyl groups by reacting with free carbon radicals. In addition, it is noted that all these reactions happen at high temperature in gaseous state. As the reaction achieves supersaturation state, small iron oxide nanocrystals yield a spherical magnetic core assembly followed by coating of carbon free radicals and carboxyl and hydroxyl groups as a shell over the magnetic core which opted a core-shell nanostructures. The slow deposition of carbon based species (free radicals, hydroxyl and carboxyl) enable uniform spherical shell over magnetic core. The three SPIONs in this work show tunable carbon coating 
which may be attributed to an impact of carbon radical formation due to amount of $\mathrm{H}_{2} \mathrm{O}_{2}$ used in the synthesis, effect of cooling temperature which simultaneously control the rate of deposition of carbon radicals over magnetic core. A uniform and well-shaped SPIONs nanostructures were formed by precise control of the reaction parameters and several attempts were made to achieve the desired nanostructures. 
Table T1. Synthesis parameters for the preparation of the SPIONs.

\begin{tabular}{|l|l|l|l|}
\hline SPIONs & $\begin{array}{l}\text { Precursor } \\
\text { concentration }(\mathbf{g m})\end{array}$ & $\begin{array}{l}\text { Amount of } \\
\mathbf{H}_{2} \mathbf{O}_{2}(\mathbf{m l})\end{array}$ & $\begin{array}{l}\text { Reaction } \\
\text { temperature }\left({ }^{\circ} \mathbf{C}\right)\end{array}$ \\
\hline Carbon composite SPIONs & 0.20 & $2.5 \mathrm{ml}$ & 220 \\
\hline $10 \mathrm{~nm}$ carbon coated SPIONs & 0.20 & $4 \mathrm{ml}$ & 220 \\
\hline $40 \mathrm{~nm}$ carbon coated SPIONs & 0.20 & $5 \mathrm{ml}$ & 220 \\
\hline
\end{tabular}

Table T2. The size of primary magnetite nanoparticles in all the three SPIONs measured using HRTEM and the mean crystalline size of magnetite nanoparticles calculated from XRD results using Scherrer equation.

\begin{tabular}{|c|c|c|}
\hline SPIONs & $\begin{array}{c}\text { Primary magnetite } \\
\text { nanoparticles average size }(\mathbf{n m})\end{array}$ & $\begin{array}{c}\text { Mean crystallites size } \\
(\mathbf{n m})\end{array}$ \\
\hline $40 \mathrm{~nm}$ carbon coated SPIONs & $8.7 \mathrm{~nm}$ & $9.5 \mathrm{~nm}$ \\
\hline $10 \mathrm{~nm}$ carbon coated SPIONs & $8.3 \mathrm{~nm}$ & $8.9 \mathrm{~nm}$ \\
\hline carbon composite SPIONs & $7.2 \mathrm{~nm}$ & $7.8 \mathrm{~nm}$ \\
\hline
\end{tabular}



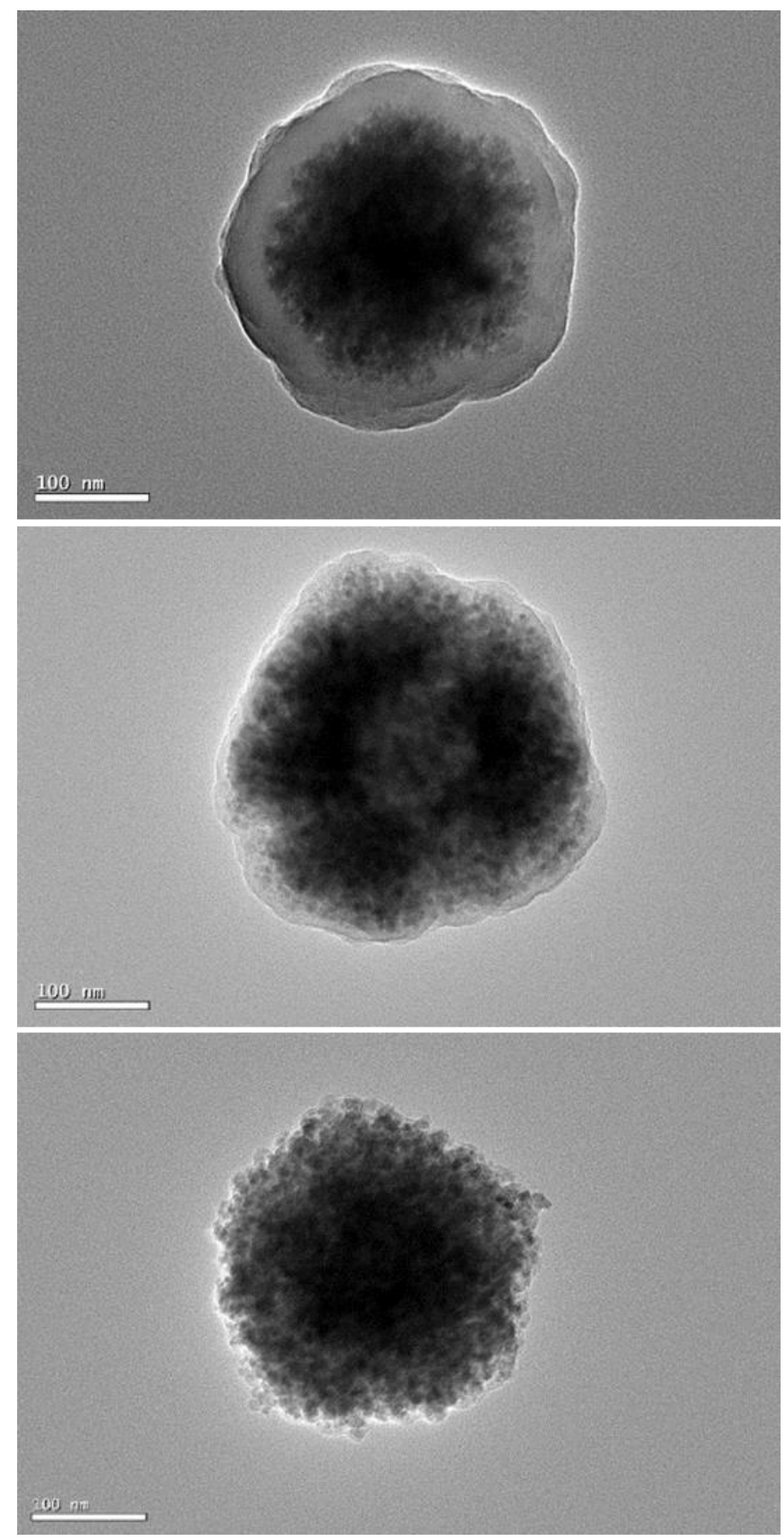

Figure S1. TEM images of SPIONs showing the particle size and morphology with the clear core-shell structures. 

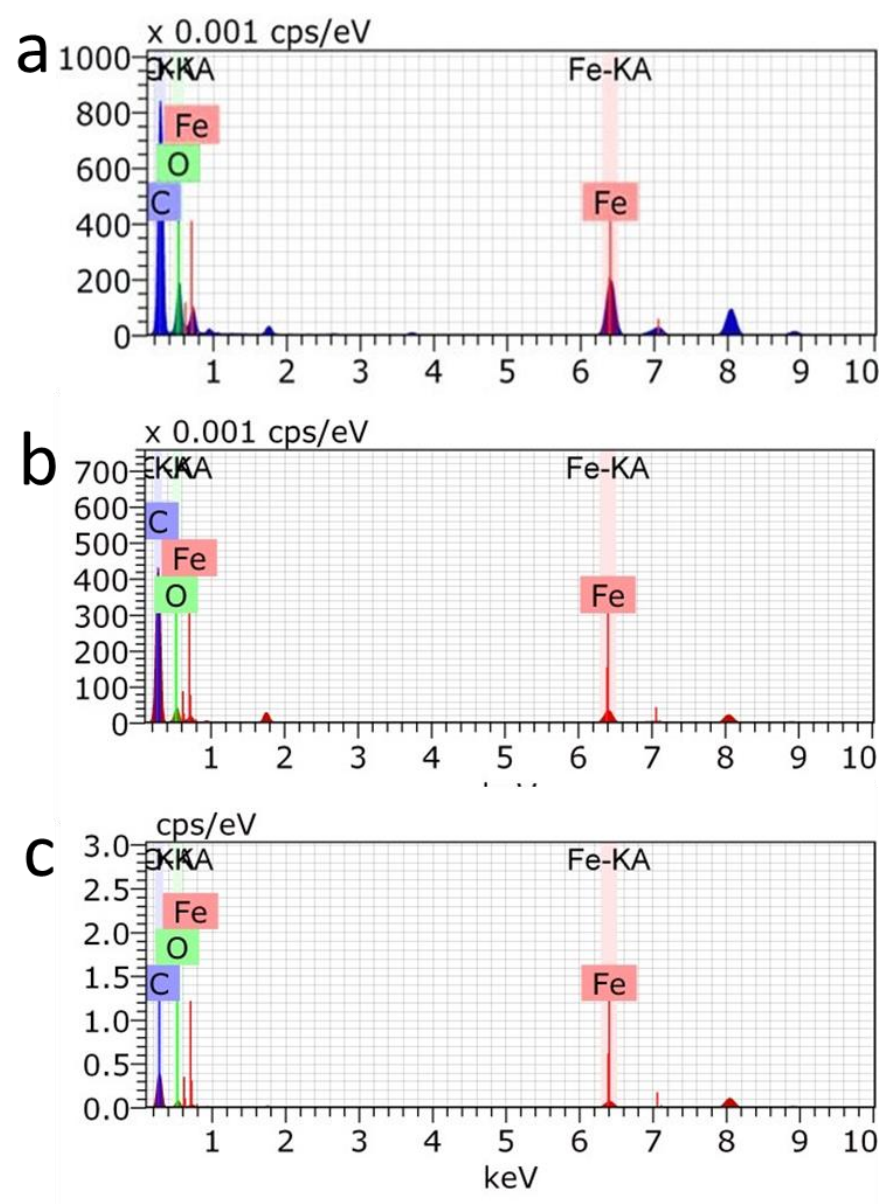

Figure S2. EDAX spectra images of (a) $40 \mathrm{~nm}$ carbon coated SPIONs (b) $10 \mathrm{~nm}$ carbon coated SPIONs and (c) carbon composite SPIONs respectively. 

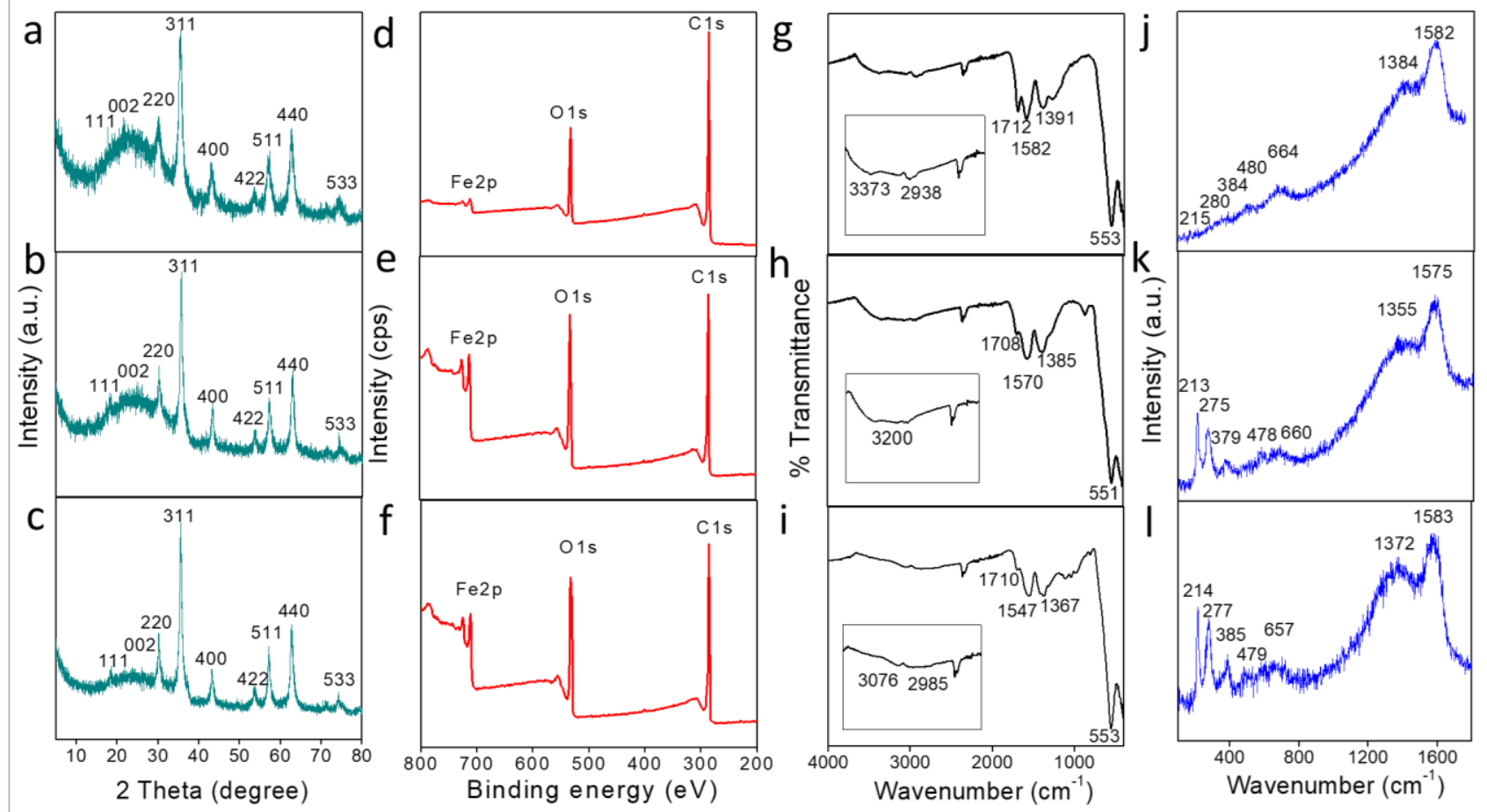

Figure S3. XRD spectra (a-c), full survey XPS spectra (d-f), FTIR spectra (g-i) and Raman spectra (j-l) of $40 \mathrm{~nm}$ carbon coated SPIONs, $10 \mathrm{~nm}$ carbon coated SPIONs and carbon composite SPIONs respectively 

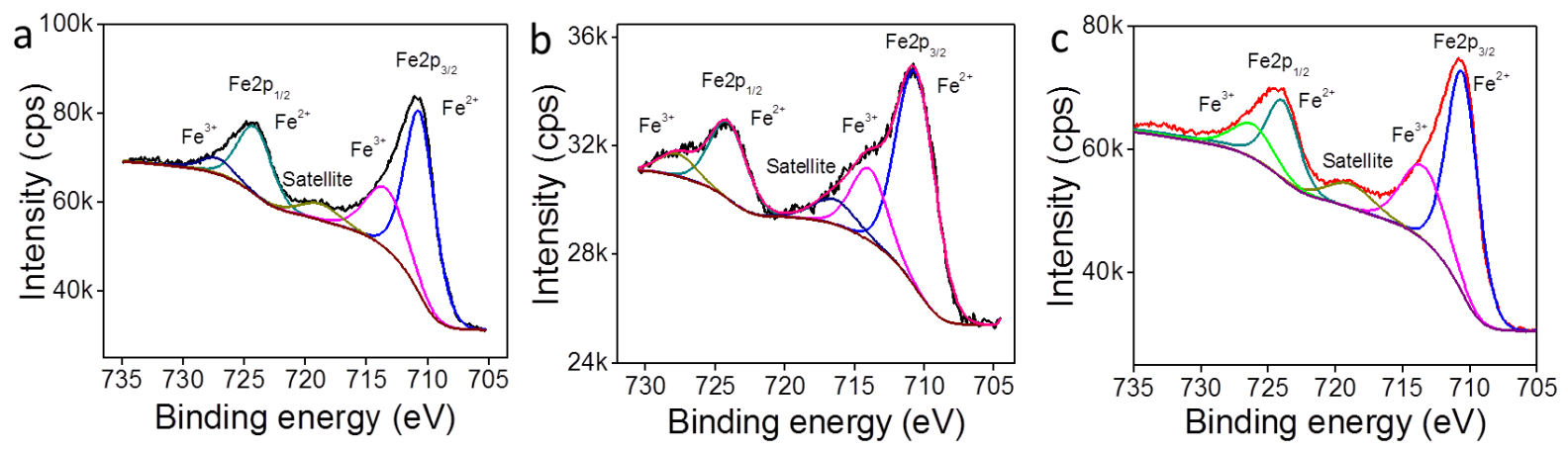

Figure S4. High-resolution deconvoluted Fe2p XPS spectra of SPIONs displayed two distinct peaks correspond to $\mathrm{Fe} 2 \mathrm{p}_{3 / 2}$ and $\mathrm{Fe} 2 \mathrm{p}_{1 / 2}$ which were deconvoluted in to four peaks along with one shakeup satellite peak. (a) $40 \mathrm{~nm}$ carbon coated SPIONs (b) $10 \mathrm{~nm}$ carbon coated SPIONs and (c) carbon composite SPIONs respectively. 

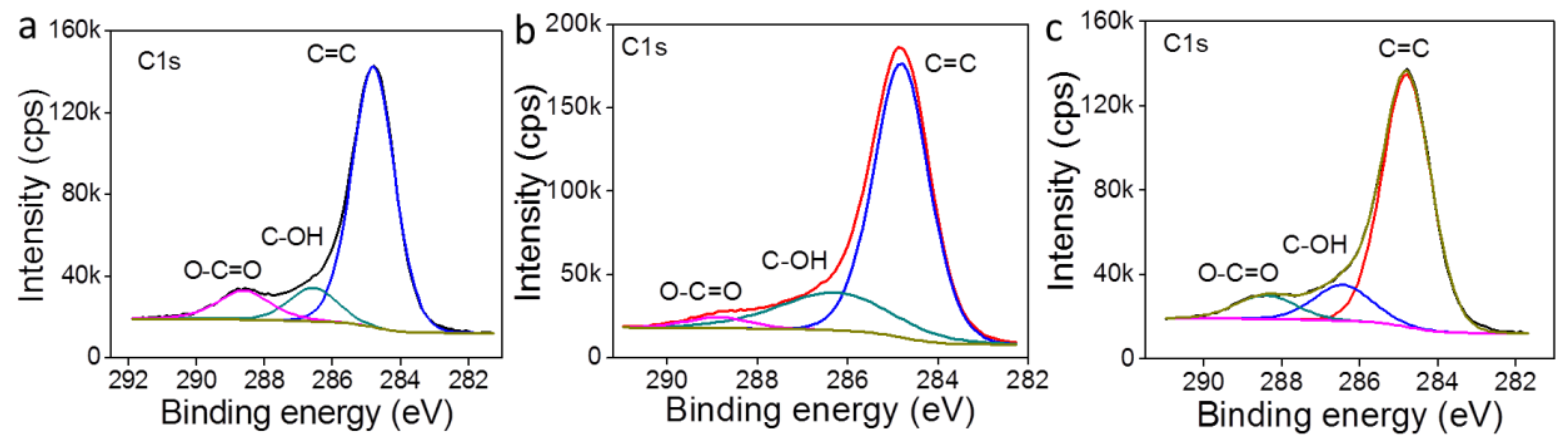

Figure S5. High-resolution deconvoluted C1s XPS spectra of SPIONs showed three peaks for $\mathrm{C}=\mathrm{C}, \mathrm{C}-\mathrm{OH}$ and $\mathrm{C}=\mathrm{O}$ respectively. (a) $40 \mathrm{~nm}$ carbon coated SPIONs (b) $10 \mathrm{~nm}$ carbon coated SPIONs and (c) carbon composite SPIONs respectively. 

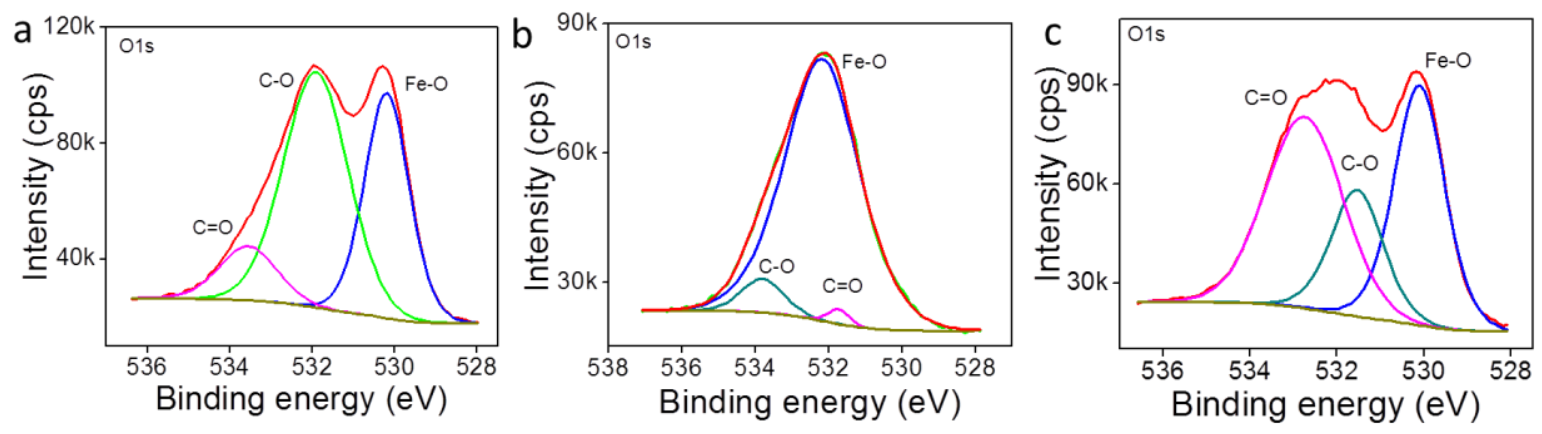

Figure S6. High-resolution deconvoluted O1s spectra of SPIONs exhibited three peaks correspond to lattice oxygen of $\mathrm{Fe}_{3} \mathrm{O}_{4}$, carbonyl oxygen in $\mathrm{O}-\mathrm{C}=\mathrm{O}$ and $\mathrm{C}-\mathrm{O}$ groups respectively. (a) $40 \mathrm{~nm}$ carbon coated SPIONs (b) $10 \mathrm{~nm}$ carbon coated SPIONs and (c) carbon composite SPIONs respectively. 


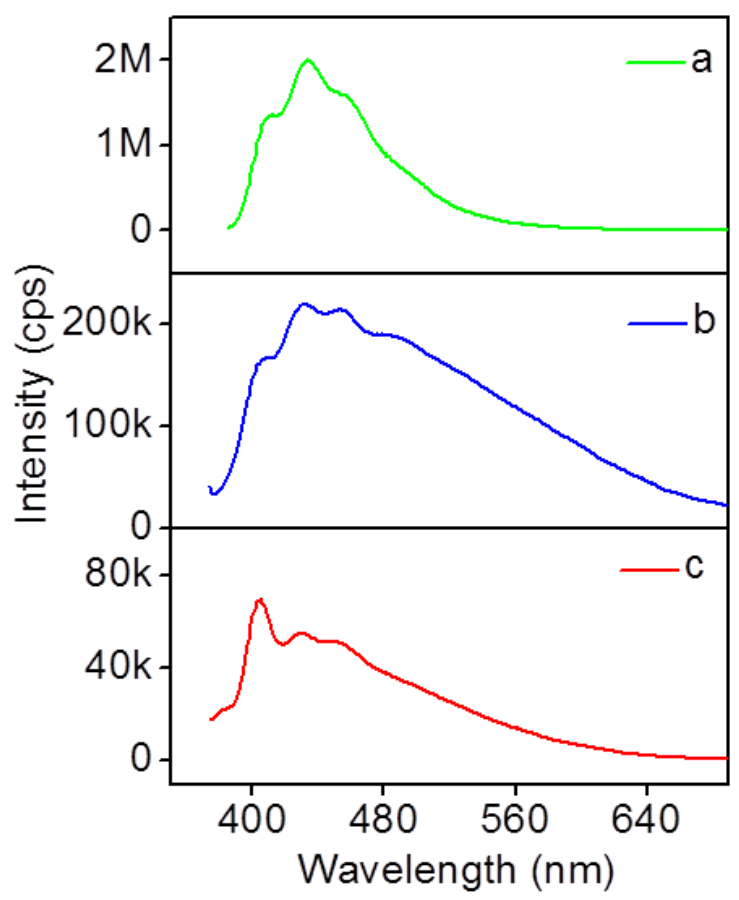

Figure S7. Emission spectra of SPIONs in bulk solution excited at $370 \mathrm{~nm}$ (a) $40 \mathrm{~nm}$ carbon coated SPIONs (b) $10 \mathrm{~nm}$ carbon coated SPIONs and (c) carbon composite SPIONs respectively. 

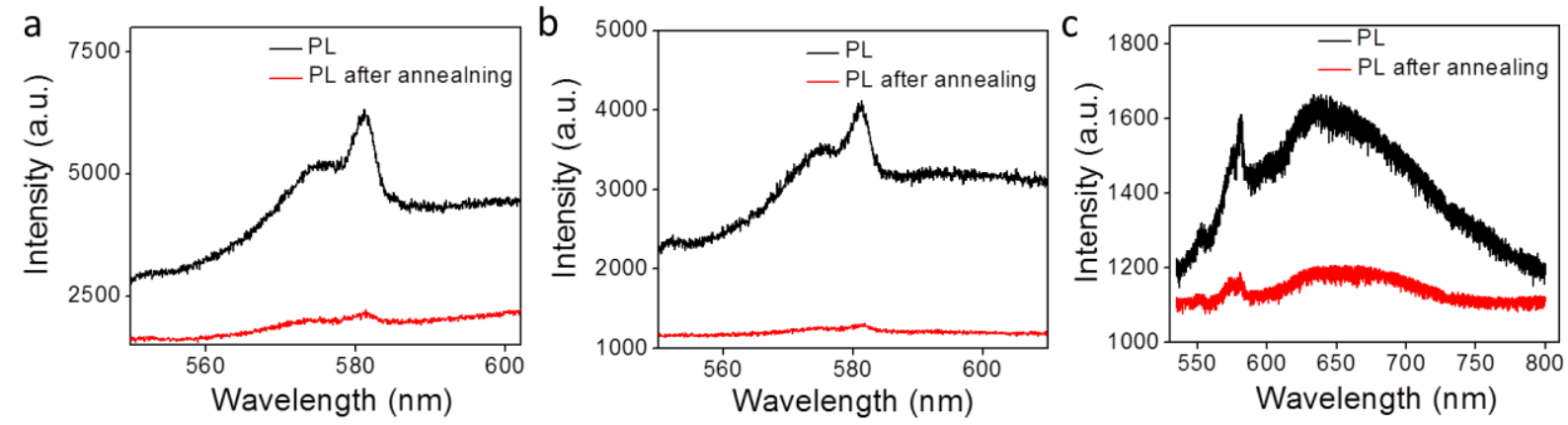

Figure S8. Photoluminescence Raman spectra of SPIONs before and after annealing the sample upto $300{ }^{\circ} \mathrm{C}$ in air which display the disappearance of luminescence peaks in annealed samples. (a) $40 \mathrm{~nm}$ carbon coated SPIONs (b) $10 \mathrm{~nm}$ carbon coated SPIONs and (c) carbon composite SPIONs respectively. 

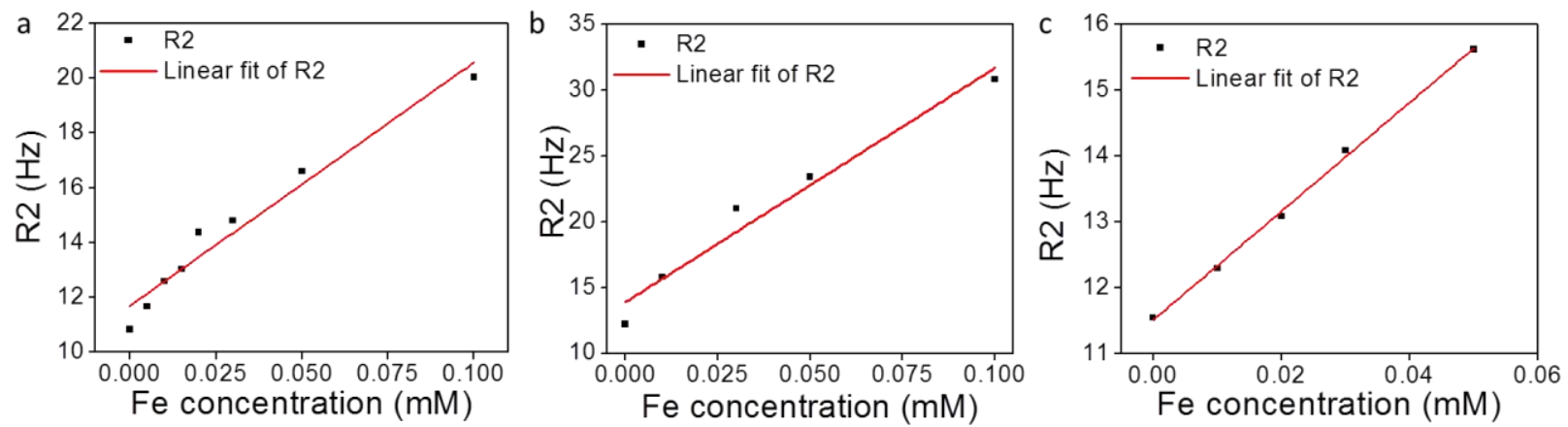

Figure S9. Plot of R2 or 1/T2 vs. Fe concentration of SPIONs. (a) $40 \mathrm{~nm}$ carbon coated SPIONs (b) $10 \mathrm{~nm}$ carbon coated SPIONs and (c) carbon composite SPIONs respectively. The red line in the plots represents a linear fit. 

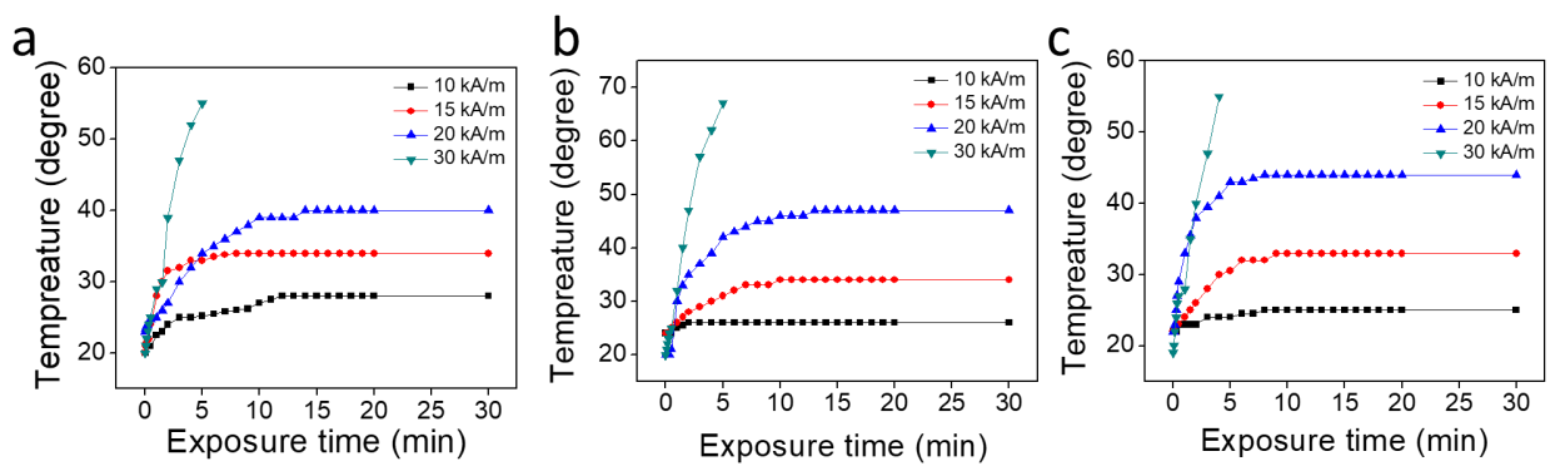

Figure S10. The heating profile curves of samples (a) $40 \mathrm{~nm}$ carbon coated SPIONs, (b) $10 \mathrm{~nm}$ carbon coated SPIONs and (c) carbon composite SPIONs $(2 \mathrm{mg} / \mathrm{ml})$ in different magnetic field amplitudes $(10,15,20$ and $30 \mathrm{kA} / \mathrm{m})$ at the frequency of $150 \mathrm{kHz}$. 
Table S3. X-ray spectroscopy analysis

\begin{tabular}{|l|l|l|l|l|l|l|l|l|l|l|l|}
\hline Samples & \multicolumn{2}{|c|}{ Fe2p3/2 } & Satellite & \multicolumn{2}{c|}{ Fe2p1/2 } & \multicolumn{3}{c|}{ O1s } & \multicolumn{3}{c|}{ C1s } \\
\hline & Fe3+ & Fe2+ & & Fe3+ & Fe2+ & Fe-O & C-O & C=O & C=C & C-OH & C=O \\
\hline $\begin{array}{l}\text { Carbon } \\
\text { composite } \\
\text { SPIONs }\end{array}$ & 726.52 & 724.01 & 718.98 & 713.73 & 710.68 & 530.12 & 531.51 & 532.77 & 284.80 & 286.47 & 288.48 \\
\hline $\begin{array}{l}10 \mathrm{~nm} \text { carbon } \\
\text { coated } \\
\text { SPIONs }\end{array}$ & 727.94 & 724.29 & 716.88 & 714.10 & 710.81 & 531.62 & 532.18 & 533.80 & 284.81 & 286.36 & 288.68 \\
\hline $\begin{array}{l}\text { 40 nm carbon } \\
\text { coated } \\
\text { SPIONs }\end{array}$ & 727.42 & 724.31 & 719.14 & 713.65 & 710.84 & 530.18 & 531.93 & 533.55 & 284.80 & 286.53 & 288.61 \\
\hline
\end{tabular}


Table S4. Functional group analysis

\begin{tabular}{|l|l|l|l|l|l|}
\hline Samples & $\begin{array}{l}\text { OH } \\
\text { stretching } \\
\left(\mathbf{c m}^{-1}\right)\end{array}$ & $\begin{array}{l}\mathbf{C = O} \\
\text { stretching } \\
\left(\mathbf{c m}^{-\mathbf{1}}\right)\end{array}$ & $\begin{array}{l}\mathbf{C = C} \\
\text { stretching } \\
\left(\mathbf{c m}^{-\mathbf{1}}\right)\end{array}$ & $\begin{array}{l}\mathbf{C}-\mathbf{H} \\
\text { bending } \\
\left(\mathbf{c m}^{-\mathbf{1}}\right)\end{array}$ & $\begin{array}{l}\text { Fe-O (metal oxide } \\
\text { linkage })\left(\mathbf{c m}^{-\mathbf{1}}\right)\end{array}$ \\
\hline $\begin{array}{l}\text { Carbon composite } \\
\text { SPIONs }\end{array}$ & 3244 & 1710 & 1552 & 1358 & 553 \\
\hline $\begin{array}{l}10 \text { nm carbon } \\
\text { coated SPIONs }\end{array}$ & 3255 & 1708 & 1570 & 1385 & 551 \\
\hline $\begin{array}{l}40 \text { nm carbon } \\
\text { coated SPIONs }\end{array}$ & 3390 & 1712 & 1582 & 1391 & 554 \\
\hline
\end{tabular}

Table S5. Magnetic hyperthermia results and SLP values.

\begin{tabular}{|l|c|c|}
\hline \multicolumn{1}{|c|}{ Sample } & Temperature $\left({ }^{\circ} \mathrm{C}\right)(20 \mathrm{kA} / \mathrm{m})$ & $\mathrm{SLP}\left(\mathrm{W} / \mathrm{gm}_{(\mathrm{Np})}\right)$ \\
\hline $10 \mathrm{~nm}$ carbon coated SPIONs & 47 & 430 \\
\hline $40 \mathrm{~nm}$ carbon coated SPIONs & 44 & 255 \\
\hline Carbon composite SPIONs & 40 & 239 \\
\hline
\end{tabular}

